\title{
Closed-Loop Communication Maintenance Management Based on Early Warning Analysis \\ Dong Gang-song
}

\author{
Information \& Telecommunication Co. of State Grid Henan Electric Power Company, Zhengzhou, \\ 450052, China \\ email:elf8650@163.com
}

\begin{abstract}
Keywords: Communication Maintenance, Early Warning Analysis, Big Data, Closed-Loop Management
\end{abstract}

\begin{abstract}
Maintenance management is an extremely important part in the work of electric power communication net, it's the key to guaranty stable operation of the network. This paper proposed that communication maintenance management based on fault early-warning analysis, used big data technology, and combined with antecedent information, machine account information and business-use information of the communication network equipment. According to the hierarchical distribution of communication network resource occupation, we can comprehensive analysis the utilization of the resources by the fiber resources, multiple section resources and channel resources, and can get an early warning to the resources' use of communication equipment and communication optical cable in real-time, tighten up the closed-loop communication maintenance management, realize the " controllable, controllability, in control" to the risk, safety and quality of operation.
\end{abstract}

\section{Introduction}

With the rapid expansion of the electric power communication business field and types, the rapid growth of the devices' number, communication networks become more and more complex and huge. Besides, with the rapid development of networks and the rapid growth of business channel, during "the 12th Five-Year Plan", the final scale of network will be 3 times comparing with now. And the number of business channel will be increase rapidly. The mode of operation will be more complex. However, the traditional network management tools only focus on a single manufacture's network operation monitoring, integrated network management tools also only focus on overall network performance, and the job of communication maintenance lacks of three-dimensional panoramic view of the multi-dimensional comprehensive approach to monitoring. Large amounts of scattered communication equipment alarm information bring the interference for the normal repair work, so the response capability in real-time monitoring is weaker. Meanwhile, the operation and maintenance of communication networks for a long time basically rely on remedial processing to the equipment failure, or network equipment maintenance cycle plan.

The former question is always in a state of passivity and delays, it can't prevent before the fault occurs; the latter's is lack of pertinence and accuracy of troubleshooting.

Power communication backbone transmission network as an object, we utilize the performance's collection and analysis to the networks and devices, and control the trends of performance's change, able to alert the communications network failures that may occur, arrange a proactive and targeted maintenance tasks, so as to eliminate the potential problem of network in advance and prevent the occurrence of the fault.

Since the 12th five-year, the State Grid Corporation unified planning, unified design and unified construction of the SG-TMS communication management system, achieved resource allocation, real-time monitoring and operation management of the backbone transmission network, and other basic applications. So it provides a solid platform for further communication network operation and maintenance management automation and intelligence. According to use the big data technology, this paper gives an early warning analysis for the communication fault and maintenance work, and provides intelligent, automatic method to aid for the making of communication maintenance decision and plan. Besides, it can improve communication efficiency and precision of maintenance 
work, and greatly reduce the workload of generation and maintenance of network topology relationship, quickly find and locate abnormal events in a communication network. So as to improve the level of communication network monitoring and management efficiency, reduce the regulators' operation pressure. It's important to improve network stability, guarantee safe operation of power grids.

\section{Early Warning Analysis Based on Big Data Technology}

According to use the big data analysis technology, and integrate the history information, account information and business information of communication network equipment, research communication network early warning analysis method based on the level model of resource utilization, so we can avoid excessive communication business to densely distribute on the same path, reduce the operation risk. In particular, the impact is very serious when a communication device failed in the path, and may have systemic safety risks. Therefore, according to the hierarchical distribution which communication network resource occupied, we can comprehensively analyze the utilization of resources from the fiber resource, reusing resource and channel resource, and it's the key which gets an early warning for the use of communication device and optical cable in real-time to improve the communication management work.

Big data technology refers to fast get valuable information technology from the huge amount of various type data. The core is big data technology to solve the problem of big data. It can be divided into 8 kinds of technology: data collection, data access, infrastructure, data processing, statistical analysis, data mining, model prediction, results presenting and so on. Big data technology mainly formed the batch processing, stream processing and interactive analysis of 3 kinds of calculation model.

Big data is not only refers to the vast amounts of information, more emphasis on human filter and processing to information. Big data processing has a lot of method, the general application of big data processing procedure can be summarized as four steps, gathering, import, and pretreatment, statistics and analysis, finally is the data mining.

The multi-processing-stage model which designed by Fayyad and others is widely accepted in currently, which is shown as Fig.1.

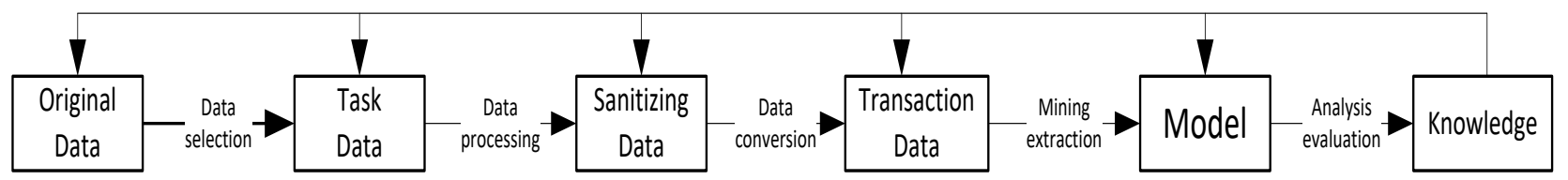

Fig.1 Multi-Processing-Stage Model

Big data research mainly as a research method or a tool of discovering new knowledge, rather than let the data itself as the research target, it is closely related with the traditional data mining methods and radically different.

\section{The Key to Communication Maintenance Management}

Horizontal Coordination, Active Docking, Enhancing Communication Maintenance Control Ability. Firstly, professional coordination, enhancing docking with professional way. Enhancing communication cooperation with professional way, holding professional specialization seminars within the department for important maintenance and plans. Implementing some important content that infrastructure, technical renovation and overhaul projects' impact on existing business and communication networks, the temporary operation mode, and the network changes after the implementation.

Secondly, "three commercial mutual", strengthening communication and consultation with the power grid control sector. Setting up "three commercial mutual" system with the power regulatory focus, and strengthening communication and coordination with the grid regulators through daily plan of electronic process sign, and consultation in weeks, months and years' plan to ensure 
communications maintenance security and developing smoothly.

Thirdly, "two figures and one chart", enhancing the level of momentous maintenance and management control. Holding a communication maintenance coordination meeting which contains construction management department, design, supervision, construction units, equipment manufacturers, schedule management and other related units. Then clear the key point, control the node of duration, and steadily put forward the progress of project. Guiding work greatly enhance the level of ability to manage and control for major overhaul according to make "two figures and one chart" (Commander and Gantt diagram, maintenance plan tables).

Vertical Linking, no Blind Angle, Implement Communication Maintenance Standardization Management. Firstly, tamping the foundation, establish a long-term safety mechanism of communication maintenance. 1) Strengthening plan management: enhance the overhaul of vertical communication, planning, reporting, strictly controlling the number and reducing the proportion of check. 2) Perfecting internal control system: implement initiation, examine and approve starts, construction, completion and materials archived that the whole process management for communication maintenance work. 3) Regularly carry out "looking back" activities: asked all units to summarize, communication maintenance security management self-issues reported to the company by e-mail.

Secondly, strengthen training, improve the overall quality of communications maintenance professionals. Through Henan communication maintenance management provides propaganda and training, application TMS system operations training, ICT training, maintenance plan and three measures and a case, standardized operation training for the mode of operation during maintenance and other training, propagate the knowledge of safety production, deepen the management of standardization, improve the overall quality of maintenance professionals, find and train professional jobs talents.

Thirdly, regular analyze, hold a monthly maintenance summary balance meeting. In order to further strength the management of communication maintenance work, adapt to the requirements for power grid construction, strengthen the linkage, improve companies' ability to work together. On 17th of the month, organize communications in the province monthly maintenance plan balance meeting, summarize and inform the situation in each unit communications maintenance about last month, analyze and discuss the communication maintenance plan about this month, strength the implementation and normative for each job, ensure the key work completed on schedule.

Formulate Norm, Grasp and Control the Process, Promote the Construction of Communication Maintenance Standardization. First, formulate norm, perfect the standardization of the communication maintenance management procedures. Completed the management and technology standard of communication maintenance, involved in the compile of power grid blackout plan management standards, fully developed operational identify, and achieved the technical standard covering the whole business, management standard covering the whole process, job standard covering the whole post on the basis of business system.

Second, control processes, implement working standardization in the communication maintenance site. According to "one process and four control"---repair work process, standard work control, resource data control, drawing data control, work comprehensive control, unified operational process, optimize operational procedure, standardize the behavior of operation, strengthen the management of closed-loop communication maintenance, and realize the " controllable, controllability, in control" to the risk, safety and quality of operation.

\section{Strengthen the Closed-loop Management of Maintenance Work}

To further strengthen the closed-loop management of communication and maintenance, carding process and rules and regulations to strengthen the communication and maintenance process safety management and control. In accordance with the State Grid Corporation of "electric power communication standard operating norms" requirements, strengthen the operation process and process control of risk analysis, unified operation process, optimize the operating procedures, 
standardize operations, realize the ", controllability, in control" to the risk, safety and quality of operation.

According to specifications combined with communication maintenance planning feature, draw up "three measures and a case of fiber cable reform", "channel switching instruction card", "communication power supply installation and commissioning work instruction", "communication line inspection card", "storage battery charging and discharging standard work instruction", the complex communication and repair process for curing into orderly operation ticket, in practice at least two checks, thus avoiding the negligence resulting in small operation link is missing problem, to further standardize the communication work in the Bureau's repair process, can effectively avoid the transfer member of human error, guarantee the entire maintenance process more orderly and controllable.

\section{Conclusion}

Electric power communication is not only the traditional power grid enterprise safety production and management of important technical support, is also the basis of smart grid automation, informatization, interacting. As communication becomes more and more prominent, we will put forward higher requirements for the security and risk management of communication networks, maintenance management work as the core communications operation will also get efficiency and quality improvements. Communication maintenance management based on fault early-warning analysis provides intelligent, automated auxiliary method for the formulation of maintenance decision-making and planning. This can effectively improve the efficiency and accuracy of maintenance work and has relevance for the power communication network and grid's security.

\section{References}

[1] Jia-Ping, Wu-Desheng, Wan-Dayang, Jia-Song. The Intelligent Maintenance Strategy Management System Based on Performance Trends and Early Warning Analysis[J]. Telecom Market, 2013(9-19):23-27.

[2] Zhang Lei, Ding Weiqiang. Research and Application on Quantitative Qisk Assessment for Communication Network Maintenance[J]. Telecommunications for Electric Power System, 2011(10), 32.

[3] ZHANG Pei. Utility experience performing probabilistic risk assessment for operational planning of electric power systems [J]. Southern Power System Technology, 2007, 1(10): 52-56.

[4] Qiu Xixia, Liu Zhicong, Zhou Yongbiao, Huang Feng. An AHP Based Evaluation Approach for Electric Power Communication Network[J]. ELECTRONIC TEST, 2013(7), 14. 